\section{Domínios de atividade física em comunidades quilombolas do sudoeste da Bahia, Brasil: estudo de base populacional}

\author{
Domains of physical activity in slave-descendant \\ communities in Southwest Bahia State, Brazil: \\ a population-based study
}

\section{Dominios de actividad física en las comunidades quilombolas del suroeste de Bahía, Brasil: un estudio basado en la población}

\author{
1 Instituto Multidisciplinar \\ em Saúde, Universidade \\ Federal da Bahia, Salvador, \\ Brasil. \\ 2 Faculdade de Medicina \\ Universidade Federal de \\ Minas Gerais, Belo Horizonte, \\ Brasil. \\ 3 Instituto de Ciências Exatas, \\ Universidade Federal de \\ Minas Gerais, Belo Horizonte, \\ Brasil. \\ Correspondência \\ V. M. Bezerra \\ Rua Rio de Contas 58 , \\ quadra 17, lote 58, Vitória da \\ Conquista, BA 45029-094, \\ Brasil. \\ vanessaenut@yahoo.com.br
}

\begin{abstract}
This study aimed to describe the prevalence of physical activity (PA) and associated factors in various domains (leisure-time, work, home, and commuting) among quilombolas (descendants of African slaves) in Bahia State, Brazil. This was a cross-sectional study of 797 individuals from 18 to 100 years of age. The study adopted a cutoff point of 150 minutes of PA per week. A hierarchical Poisson model was used. The highest prevalence of PA was at work (42.1\%), followed by the home environment (39.3\%), commuting (35.5\%), and leisure time (13.1\%). PA at work was associated with male gender, lower age, higher schooling, and consumption of alcohol and fruits. PA in the household domain was associated with female gender, lower age, marital status (married), and negative self-rated health. In commuting, PA was associated with male gender and lower age bracket, and during leisure time with safety, male gender, lower age, and higher schooling. The study concludes that this slavedescendant community displays a profile of $P A$ that is characteristic of rural groups (more active at work, with little leisure-time activity). The determinants of PA were similar to those seen in urban groups.
\end{abstract}

Motor Activity; Vulnerable Groups; African Continental Ancestry Group; Risk Factors
Vanessa Moraes Bezerra 1,2 Amanda Cristina de Souza Andrade 2 Cibele Comini César 3 Waleska Teixeira Caiaffa ${ }^{2}$

\section{Resumo}

Este estudo objetivou descrever a prevalência e os fatores associados à atividade física (AF) nos domínios lazer, trabalho, doméstico e deslocamento, em residentes quilombolas. Estudo transversal, com amostra de 797 indivíduos, na faixa etária de 18 a 100 anos, para o qual se adotou o ponto de corte de 150 minutos de AF semanal e utilizou-se o modelo de Poisson com entrada hierárquica de variáveis. A maior prevalência de AF foi no domínio trabalho (42,1\%), seguido do doméstico (39,3\%), deslocamento (35,5\%) e lazer (13,1\%). Sexo masculino, menor faixa etária, maior escolaridade, consumo de álcool e frutas foram associados com o domínio AF no trabalho; sexo feminino, menor faixa etária, ser casado e percepção negativa da saúde, com o doméstico; sexo masculino e menor faixa etária, com o domínio deslocamento; segurança, sexo masculino, menor faixa etária e maior escolaridade, com o lazer. Conclui-se que os quilombolas apresentaram perfil de AF característico de grupos rurais, sendo mais ativos no trabalho e pouco ativos no lazer. Os determinantes da AF foram semelhantes aos observados em grupos urbanos.

Atividade Motora; Comunidades Vulneráveis; Grupo com Ancestrais do Continente Africano; Fatores de Risco 


\section{Introdução}

A adoção da prática regular de atividade física (AF) é um importante comportamento para prevenção e tratamento não medicamentoso de doenças crônicas não transmissíveis (DCNT) 1,2. Além de aspectos individuais e sociais, os ambientais também têm sido associados aos níveis de AF, uma vez que o contexto físico e social em que pessoas estão inseridas podem ser determinantes da prática dessas atividades 3,4 . Essa situação explica as recomendações atuais para prática regular de AF acumulada em diferentes contextos: lazer, ambiente de trabalho, ambiente doméstico e deslocamento 4,5 .

Estudos epidemiológicos indicam que grande parcela da população não atende às recomendações atuais quanto aos níveis de $\mathrm{AF}$ 2,6. Tal quadro é frequentemente observado em grupos economicamente desfavorecidos, os quais geralmente apresentam menor frequência de $\mathrm{AF} 7$, mas especificamente no âmbito do lazer 8,9 . A falta de dinheiro, de informação e de ambientes favoráveis podem influenciar na ocorrência de inatividade física nessas populações 7 .

Inseridas no contexto das populações rurais e economicamente desfavorecidas, estão as comunidades quilombolas, conceituadas como grupos étnico-raciais de definição autoatribuível, com trajetória histórica própria, dotadas de relações territoriais específicas e com presunção de ancestralidade negra relacionada com a resistência à opressão histórica sofrida 10 . As situações de vulnerabilidade social nessas populações podem ser historicamente explicadas tanto pelo período de escravidão no Brasil no século XIX, quanto pelas baixas condições socioeconômicas 11 e elevadas prevalências de DCNT 12,13.

O plano de ações para enfrentamento das DCNT 14, lançado em 2011 pelo Ministério da Saúde, aborda estratégias para prevenir e controlar essas doenças no Brasil nos próximos dez anos. Entre os seus principais objetivos, destaca-se a promoção da saúde por meio da AF, com atenção especial a grupos vulneráveis, como os quilombolas.

Diante dessa perspectiva, este estudo teve por objetivo descrever a prevalência de AF e identificar fatores associados a ela nos domínios lazer, trabalho, doméstico e deslocamento, em comunidades quilombolas da região sudoeste da Bahia, Brasil.

\section{Métodos}

Os dados deste estudo derivam de um inquérito domiciliar intitulado Projeto COMQUISTA: $\mathrm{Co}$ - munidades Quilombolas de Vitória da Conquista - BA. Avaliação de Saúde e seus Condicionantes 11, realizado no período de setembro a outubro de 2011. Trata-se de estudo transversal de base populacional, que avaliou comunidades quilombolas localizadas na região sudoeste da Bahia (Região Nordeste do Brasil).

O Município de Vitória da Conquista registra 25 comunidades quilombolas certificadas pela Fundação Palmares, sediadas em cinco distritos da região. Os critérios de inclusão adotados foram possuir certificação pela Fundação Palmares e ter mais de cinquenta famílias na comunidade. A população pesquisada foi estimada em 2.935 indivíduos adultos oriundos de dez comunidades quilombolas que atenderam aos critérios de inclusão. Para o cálculo do tamanho amostral, foi considerada uma prevalência de $50 \%$, em face da heterogeneidade dos eventos mensurados no projeto principal; precisão de $5 \%$; intervalo de $95 \%$ de confiança (IC95\%); efeito de desenho = 2 e $30 \%$ de perdas, totalizando uma amostra de 884 indivíduos adultos 11 .

O delineamento amostral foi realizado em dois estágios: (1) seleção aleatória de uma comunidade quilombola em cada distrito, com probabilidade proporcional à população da comunidade; quando o distrito tinha somente uma comunidade, esta foi selecionada; (2) seleção aleatória dos domicílios, de acordo com a distribuição proporcional destes, por distrito. Nos domicílios selecionados, todos os residentes com 18 anos ou mais foram convidados a participar da pesquisa.

Realizaram-se entrevistas individuais, utilizando-se computador portátil (HP Pocket Rx5710, Hewlett-Packard Development Company, Estados Unidos) para registro e armazenamento dos dados. Foi adotado o questionário semiestruturado da Pesquisa Nacional de Saúde (PNS) 15, em versão adaptada para a população quilombola. As questões sobre AF da PNS foram construídas com base no questionário do VIGITEL (Vigilância de Fatores de Risco e Proteção para Doenças Crônicas por Inquérito Telefônico) 16. Adaptações foram realizadas com o objetivo de facilitar a compreensão e retratar a realidade de uma comunidade quilombola, sem alterar a estrutura do questionário. No bloco de AF, foram feitas adaptações em termos de vocabulário e/ ou opções de respostas; atividades como jogar tênis e fazer hidroginástica foram substituídas por capoeira e cavalgar (como forma de lazer). Também foi inserido o termo "baba", expressão que caracteriza o futebol na região 11 .

O instrumento adotado contém 14 questões referentes à frequência de dias e tempo gasto em AF nos domínios: lazer (modalidade do exercí- 
cio físico ou esporte e intensidade da atividade), trabalho (carregar peso, fazer esforço intenso ou caminhar bastante), deslocamento (caminhar ou andar de bicicleta para o trabalho e/ou atividades habituais) e doméstico (realizar faxina, carregar peso ou fazer esforço intenso).

A variável resposta foi a prática de $\mathrm{AF}$ em seus quatro domínios (lazer, trabalho, doméstico e deslocamento), cujo cálculo considerou a multiplicação da frequência semanal (dias) pela duração média (minutos) da prática de caminhada e outras AF moderadas e vigorosas. O tempo despendido em atividades vigorosas foi multiplicado por dois. Apenas atividades desempenhadas por pelo menos dez minutos contínuos foram validadas 6 . Foram considerados ativos os indivíduos que praticavam 150 minutos ou mais de atividade física/semana em cada domínio avaliado 5,6,16.

O coeficiente de correlação intraclasse (CCI) e estatística kappa foram calculados com base nas respostas dos entrevistados que participaram do estudo de confiabilidade teste-reteste $(\mathrm{n}=32)$. Verificou-se reprodutibilidade satisfatória do questionário, com nível de estabilidade das respostas dos domínios de AF classificado como provável a quase perfeito, segundo os pontos de corte sugeridos por Landis \& Koch 17 (CCIC $=91$,95 para domínio do lazer; CCI $=98,83$ para $\mathrm{AF}$ no trabalho; $\mathrm{CCI}=78,65$ para $\mathrm{AF}$ no deslocamento; e $\mathrm{CCI}=39,79$ para $\mathrm{AF}$ no domínio doméstico). Os coeficientes de kappa variaram entre 44,4 e 100, indicando concordância de moderada a quase perfeita 17 .

As variáveis independentes foram agrupadas em quatro blocos de acordo com um modelo conceitual hierárquico proposto para AF (Figura 1), adaptado de Dumith 18. No bloco mais distal (primeiro bloco), estavam variáveis relacionadas à percepção individual do entorno físico e social, no que diz respeito aos seguintes fatores do ambiente: presença de área de lazer, estética do lugar (presença de lixo ou entulho). Quanto à percepção de segurança, foi questionado se o indivíduo se sentia seguro ao caminhar na vizinhança durante dia e noite.

O segundo bloco foi constituído por variáveis demográficas e socioeconômicas, tais como: sexo, idade, estado conjugal, escolaridade (nunca estudou, 1 a 4 e 5 ou mais anos de estudo) e classes econômicas E, D, C/B (Associação Brasileira de Empresas de Pesquisa. Critérios de classificação econômica no Brasil. http:/ / www.abep. org/novo / filegenerate.ashx?id=257, acessado em 21/Mar/2012).

Variáveis comportamentais compuseram o terceiro bloco. O tabagismo foi categorizado em nunca fumou, fumante e ex-fumante. O consu- mo de álcool foi considerado de risco quando o etanol ingerido foi acima de $30 \mathrm{~g} /$ dia para homens e acima de 15g/dia para mulheres 19. Para a dieta, foi avaliada a frequência diária do consumo de frutas e legumes/verduras 12 , dicotomizadas em não consome e consome pelo menos uma vez no dia.

No bloco mais proximal (quarto bloco), foram investigados fatores de saúde/doença. Medidas de peso e altura foram aferidas para o cálculo do índice de massa corporal (IMC); cuja classificação obedeceu aos seguintes pontos de corte, em kg/m²: < 18,5 (baixo peso), $\geq 18,5$ e $<25$ (eutrófico), $\geq 25$ e $<30$ (sobrepeso) e $\geq 30$ (obesidade), para adultos 20 ; $\leq 22$ (baixo peso), $>22 \mathrm{e}<$ 27 (eutrófico) e $\geq 27$ (sobrepeso), para idosos 21 . A circunferência da cintura (CC) também foi aferida, sendo considerada aumentada quando o valor encontrado foi $\geq 94 \mathrm{~cm}$ para homens $\mathrm{e} \geq$ $80 \mathrm{~cm}$ para mulheres e muito aumentada quando o valor foi $\geq 102 \mathrm{~cm} \mathrm{e} \geq 88 \mathrm{~cm}$ para homens e mulheres, respectivamente 20 .

Diabetes e artrite foram condições autorreferidas, isto é, o indivíduo relatava se algum médico já lhe havia dado o diagnóstico da doença. A pressão arterial foi aferida considerando-se três medidas (com intervalo de um minuto entre cada aferição), sendo utilizado o esfigmomanômetro digital da marca Omron modelo HEM-742 (OMRON Corp., São Paulo, Brasil) 12. As aferições foram realizadas ao final da entrevista, visando a garantir que os indivíduos estivessem em repouso: sentados, pernas descruzadas, pés apoiados no chão, dorso recostado na cadeira e relaxado, com o braço esquerdo apoiado sobre a mesa à altura do coração. Certificou-se de que os indivíduos não estavam com a bexiga cheia, não haviam praticado exercícios físicos há pelo menos $60 \mathrm{mi}-$ nutos, não haviam ingerido bebidas alcoólicas, café ou alimento e não haviam fumado nos 30 minutos anteriores 22 . Para análise, foi realizada a média das duas últimas aferições, sendo considerados hipertensos indivíduos que apresentaram pressão arterial sistólica $\geq 140 \mathrm{mmHg}$ e/ou pressão arterial diastólica $\geq 90 \mathrm{mmHg}$ e/ou relataram uso de medicamentos anti-hipertensivos 23 . A autopercepção de saúde foi dicotomizada em positiva (muito boa, boa) e negativa (regular, ruim e muito ruim) 24 .

Foram estimadas prevalências de AF nos quatro domínios e seus respectivos IC95\%. Foram realizadas análises descritivas com cálculos de proporções e de prevalências de AF dos diferentes domínios de acordo com cada uma das variáveis independentes. Diferenças nas proporções foram avaliadas pelo teste qui-quadrado de Pearson ou exato de Fisher, quando indicado. $\mathrm{Na}$ análise ajustada, foram estimadas razões de 


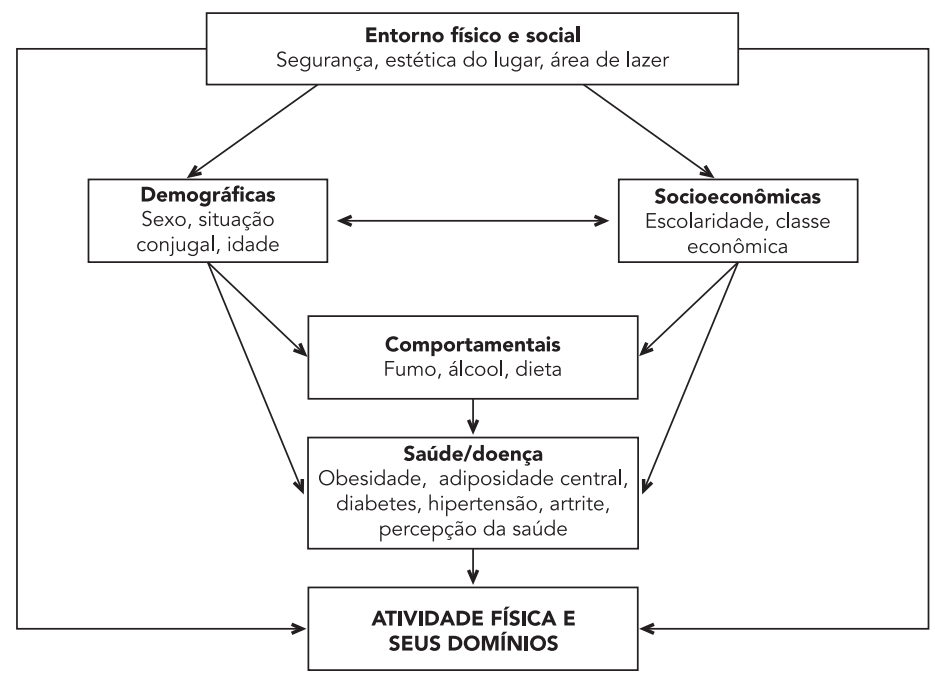

prevalência e IC95\% por meio de regressão de Poisson, com variância robusta. As variáveis que apresentaram valor de $\mathrm{p}<0,20$ na análise bivariada, em pelo menos um dos domínios de $\mathrm{AF}$, foram inseridas no modelo multivariado.

A análise multivariada foi realizada por meio da entrada hierárquica 25 em blocos de variáveis, na seguinte ordem: entorno físico e social; demográficas e socioeconômicas; comportamentais e saúde/doença. As variáveis dos blocos mais distais permaneceram como fatores de ajuste para as os blocos hierarquicamente inferiores. Para a interpretação dos resultados, a identificação de associação estatisticamente significativa (valor de $\mathrm{p} \leq 0,05$ ) entre um determinado fator em estudo e a AF após ajuste para os potenciais fatores do mesmo bloco e daqueles hierarquicamente superiores indica a existência de um efeito independente, próprio do fator. A comparação entre modelos foi feita pelo critério de Akaike (AIC), e a adequação dos modelos foi verificada por meio do teste de bondade de ajuste.

Como todos os residentes no domicílio com 18 anos ou mais foram entrevistados, avaliouse o efeito de agrupamento intradomiciliar, utilizando-se a estatística DEFT (design effect). Uma vez que não foi evidenciado efeito de cluster, optou-se pelo modelo que não considera o efeito de agrupamento.

No estudo principal, foi observada uma perda de $15,5 \%$ da amostra, cujo principal motivo foi o fato de não se encontrarem os indivíduos selecionados após três visitas ao seu domicílio $(82,2 \%)$. A perda foi significativamente maior entre aqueles do sexo masculino e com idade entre 18 a 34 anos 11. Contudo, ao realizar a ponderação das perdas diferenciais usando fatores de calibração, não foram observadas diferenças significativas nas estimativas de ponto das prevalências de AF. Desse modo, as análises foram conduzidas sem considerar a ponderação. Todas as análises foram realizadas utilizando-se o software Stata versão 12 (Stata Corp., College Station, Estados Unidos).

O projeto foi aprovado pelos Comitês de Ética em Pesquisa da Faculdade São Francisco de Barreiras (CAAE 0118.0.066.000-10) e da Universidade Federal de Minas Gerais (CAAE 0118.0.066.203-10). A participação dos indivíduos foi voluntária, após assinatura do Termo de Consentimento Livre e Esclarecido.

\section{Resultados}

Entre os 797 indivíduos que participaram da pesquisa, mais da metade tinha idade acima de 40 anos, 54,3\% eram do sexo feminino e $61,4 \%$, casados. A maioria ( $80 \%$ ) da população foi classificada nas categorias D e E para classes econômicas. Quanto à escolaridade, apenas 27,7\% estudaram por, pelo menos, cinco anos, 37,7\% tinham de um a quatro anos de estudo e $34,6 \%$ nunca haviam frequentado a escola. 
A maior prevalência de AF foi de $42,1 \%$ (IC95\%: 38,6; 45,5) no domínio do trabalho, seguido de 39,3\% (IC95\%: 35,9; 42,7) no doméstico, 35,5\% (IC95\%: 32,1; 38,8) no deslocamento e $13,1 \%$ (IC95\%: 10,8; 15,5) no domínio do lazer. As comparações entre sexo apresentaram diferenças significativas em todos os domínios avaliados: os homens foram mais ativos nos domínios do lazer, trabalho e deslocamento, já as mulheres foram mais ativas no domínio doméstico (Figura 2).

Na análise bivariada (Tabela 1), a única variável do entorno físico e social associada à $\mathrm{AF}$ foi segurança na vizinhança com o domínio do lazer (valor de $\mathrm{p}=0,049$ ). No bloco das variáveis demográficas e socioeconômicas, a maioria se associou aos diferentes domínios de AF, com exceção de classe econômica, que se associou apenas com os domínios do lazer e doméstico, e estado civil, que não foi associado ao domínio do deslocamento. Quanto às variáveis comportamentais, fumo e consumo de álcool associaram-se a todos os domínios, com exceção do lazer. Consumo de legumes/verduras associou-se à AF no lazer e trabalho; consumo de frutas, somente com este último. Para os fatores saúde/doença, a circunferência da cintura associou-se à AF de todos os domínios. Autopercepção de saúde associou-se apenas com o domínio doméstico; diabetes, com o trabalho. Com relação à hipertensão, esta não se associou à AF apenas no domínio doméstico.

Na Tabela 2, estão os resultados de análises ajustadas hierárquicas. As variáveis associadas aos diferentes domínios de AF foram as seguintes: (a) no domínio do lazer - segurança na vizinhança, sexo masculino, menor faixa etária e maior escolaridade; (b) no domínio do trabalho - sexo masculino, menor faixa etária, maior escolaridade, consumo de álcool e frutas; (c) no domínio doméstico - sexo feminino, menor faixa etária, estado civil e autopercepção negativa da saúde; (d) no domínio deslocamento - sexo masculino e menor faixa etária.

Todos os modelos apresentaram ajuste adequado (valor de $\mathrm{p} \geq 0,05$ ), e a estimativa do AIC diminuiu com o ajuste dos blocos de variáveis.

\section{Discussão}

Trata-se de um primeiro estudo de base populacional realizado em comunidades quilombolas, o qual avaliou a AF em seus diferentes domínios e fatores associados. A maior prevalência de AF observada foi no domínio do trabalho e a menor, no lazer.

A prática regular de $\mathrm{AF}$ tem se mostrado como um componente fundamental para o desenvolvimento de aspectos positivos relacionados à saúde. Entretanto, estudos têm indicado baixos níveis desse comportamento na população 2,26. Semelhante ao observado em pesquisas que avaliaram populações rurais 9,27, os quilombolas estudados apresentaram baixo percentual de AF no lazer.

Quando comparada com adultos de comunidades rurais do Estado de Minas Gerais 9, a população quilombola foi mais ativa no domínio do trabalho, sendo a prestação de serviço em lavouras de café a principal atividade realizada por este grupo. Essa atividade laboral pode explicar a menor prevalência de AF no domínio

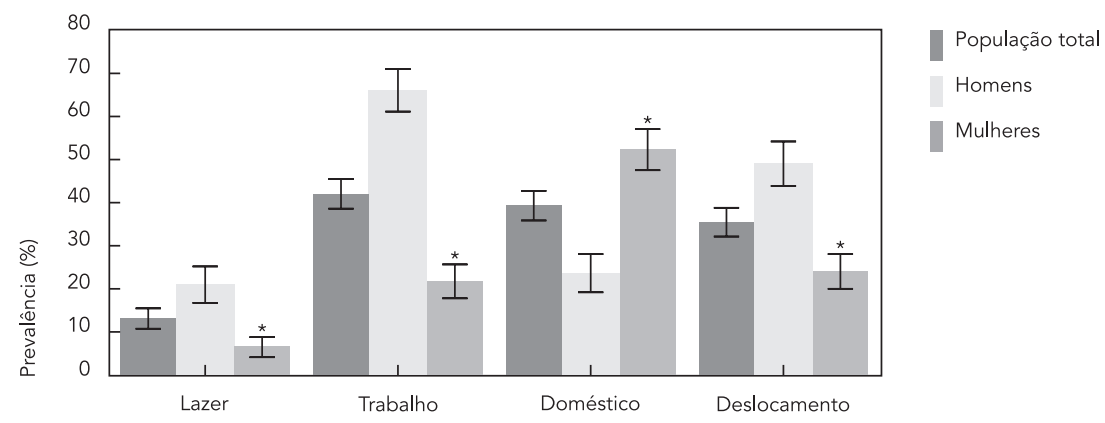

* Diferente do sexo masculino (valor de $p<0,001)$. 
Tabela 1

Distribuição da amostra e prevalência de atividade física nos domínios lazer, trabalho, doméstico e deslocamento, segundo variáveis do entorno físico e social, demográficas e socioeconômicas, comportamentais e de saúde/doença.

\begin{tabular}{|c|c|c|c|c|c|c|c|c|c|}
\hline \multirow[t]{2}{*}{ Variável } & \multirow{2}{*}{$\begin{array}{c}\text { População total } \\
\text { n (\%) }\end{array}$} & \multicolumn{2}{|c|}{ Lazer } & \multicolumn{2}{|c|}{ Trabalho } & \multicolumn{2}{|c|}{ Doméstico } & \multicolumn{2}{|c|}{ Deslocamento } \\
\hline & & $\%$ & $\begin{array}{l}\text { Valor } \\
\text { de } p\end{array}$ & $\%$ & $\begin{array}{l}\text { Valor } \\
\text { de } p\end{array}$ & $\%$ & $\begin{array}{l}\text { Valor } \\
\text { de } p\end{array}$ & $\%$ & $\begin{array}{l}\text { Valor } \\
\text { de p }\end{array}$ \\
\hline \multicolumn{10}{|l|}{ Bloco 1: entorno físico e social } \\
\hline \multicolumn{10}{|l|}{ Lixo na vizinhança } \\
\hline Sim & $171(21,6)$ & 15,2 & 0,389 & 46,5 & 0,208 & 44,9 & 0,087 & 34,8 & 0,331 \\
\hline Não & $619(78,4)$ & 12,7 & & 41,1 & & 37,6 & & 38,8 & \\
\hline \multicolumn{10}{|l|}{ Área de lazer } \\
\hline Sim & $209(26,4)$ & 16,5 & 0,120 & 45,6 & 0,245 & 40,7 & 0,626 & 39,4 & 0,181 \\
\hline Não & $582(73,6)$ & 12,1 & & 41,0 & & 38,7 & & 34,3 & \\
\hline \multicolumn{10}{|l|}{ Segurança na vizinhança } \\
\hline Sim & $532(67,3)$ & 14,9 & 0,049 & 42,7 & 0,738 & 36,9 & 0,062 & 33,9 & 0,168 \\
\hline Não & $258(32,7)$ & 9,8 & & 41,4 & & 43,9 & & 38,9 & \\
\hline \multicolumn{10}{|c|}{ Bloco 2: demográficas e socioeconômicas } \\
\hline \multicolumn{10}{|l|}{ Sexo } \\
\hline Masculino & $364(45,7)$ & 21,0 & $<0,001$ & 66,1 & $<0,001$ & 23,7 & $<0,001$ & 49,0 & $<0,001$ \\
\hline Feminino & $433(54,3)$ & 6,5 & & 21,8 & & 52,3 & & 24,1 & \\
\hline \multicolumn{10}{|l|}{ Idade (anos) } \\
\hline $18-29$ & $201(25,2)$ & 26,8 & $<0,001$ & 41,4 & $<0,001$ & 43,7 & $<0,001$ & 30,0 & $<0,001$ \\
\hline $30-39$ & $166(20,8)$ & 13,9 & & 44,9 & & 43,9 & & 41,6 & \\
\hline $40-49$ & $149(18,7)$ & 6,0 & & 53,1 & & 42,2 & & 45,0 & \\
\hline $50-59$ & $104(13,1)$ & 10,7 & & 57,7 & & 47,1 & & 43,3 & \\
\hline 60 ou mais & $177(22,2)$ & 4,5 & & 21,4 & & 22,8 & & 23,3 & \\
\hline \multicolumn{10}{|l|}{ Estado conjugal } \\
\hline Nunca foi casado & $187(23,5)$ & 20,0 & 0,005 & 45,7 & $<0,001$ & 33,9 & 0,001 & 37,6 & 0,335 \\
\hline Casado/Em união & $489(61,4)$ & 11,5 & & 44,7 & & 44,2 & & 36,1 & \\
\hline Separado/Divorciado/Viúvo & $121(15,2)$ & 9,2 & & 25,8 & & 28,0 & & 29,8 & \\
\hline \multicolumn{10}{|l|}{ Escolaridade (anos) } \\
\hline Nunca estudou & $274(34,6)$ & 5,9 & $<0,001$ & 30,6 & $<0,001$ & 29,4 & $<0,001$ & 28,0 & 0,005 \\
\hline $1-4$ & $299(37,7)$ & 10,4 & & 47,0 & & 46,2 & & 40,8 & \\
\hline 5 ou mais & $219(27,7)$ & 26,2 & & 49,1 & & 42,1 & & 37,0 & \\
\hline \multicolumn{10}{|l|}{ Classe econômica } \\
\hline Classe E & $275(34,8)$ & 10,3 & 0,017 & 38,6 & 0,304 & 32,7 & 0,027 & 37,8 & 0,439 \\
\hline Classe D & $401(50,8)$ & 13,0 & & 43,8 & & 42,8 & & 35,5 & \\
\hline Classe C e B2 & $114(14,4)$ & 21,1 & & 45,5 & & 42,0 & & 31,0 & \\
\hline \multicolumn{10}{|l|}{ Bloco 3: comportamentais } \\
\hline \multicolumn{10}{|l|}{ Fumo } \\
\hline Nunca fumou & $431(54,1)$ & 15,7 & 0,072 & 32,6 & $<0,001$ & 44,0 & 0,010 & 28,1 & $<0,001$ \\
\hline Fumante & $156(19,6)$ & 10,3 & & 56,1 & & 30,7 & & 45,5 & \\
\hline Ex-fumante & $210(26,3)$ & 10,0 & & 51,0 & & 36,4 & & 43,1 & \\
\hline \multicolumn{10}{|l|}{ Álcool (consumo de risco) } \\
\hline Sim & $104(13,1)$ & 19,2 & 0,050 & 65,4 & $<0,001$ & 27,0 & 0,007 & 50,0 & 0,001 \\
\hline Não & $688(86,9)$ & 12,2 & & 38,5 & & 41,2 & & 33,5 & \\
\hline \multicolumn{10}{|l|}{ Consumo de legumes/verduras } \\
\hline Consome & $669(84,6)$ & 14,1 & 0,045 & 44,1 & 0,006 & 40,1 & 0,413 & 36,0 & 0,498 \\
\hline Não consome & $122(15,4)$ & 7,4 & & 30,5 & & 36,1 & & 32,8 & \\
\hline Consumo de frutas & & & & & & & & & \\
\hline Consome & $635(80,6)$ & 13,6 & 0,569 & 45,0 & 0,004 & 40,7 & 0,189 & 37,0 & 0,148 \\
\hline Não consome & $153(19,4)$ & 11,8 & & 32,0 & & 34,9 & & 30,7 & \\
\hline
\end{tabular}

(continua) 
Tabela 1 (continuação)

\begin{tabular}{|c|c|c|c|c|c|c|c|c|c|}
\hline \multirow[t]{2}{*}{ Variável } & \multirow{2}{*}{$\begin{array}{c}\text { População total } \\
\text { n (\%) }\end{array}$} & \multicolumn{2}{|c|}{ Lazer } & \multicolumn{2}{|c|}{ Trabalho } & \multicolumn{2}{|c|}{ Doméstico } & \multicolumn{2}{|c|}{ Deslocamento } \\
\hline & & $\%$ & $\begin{array}{l}\text { Valor } \\
\text { de } p\end{array}$ & $\%$ & $\begin{array}{l}\text { Valor } \\
\text { de } p\end{array}$ & $\%$ & $\begin{array}{l}\text { Valor } \\
\text { de } p\end{array}$ & $\%$ & $\begin{array}{l}\text { Valor } \\
\text { de } p\end{array}$ \\
\hline \multicolumn{10}{|l|}{ Bloco 4: saúde/doença } \\
\hline \multicolumn{10}{|l|}{ Categorias de IMC } \\
\hline Baixo peso/Eutrófico & $484(63,1)$ & 14,3 & 0,266 & 45,4 & 0,072 & 35,4 & 0,002 & 35,6 & 0,319 \\
\hline Sobrepeso & $234(30,5)$ & 12,9 & & 42,4 & & 44,4 & & 39,3 & \\
\hline Obeso & $49(6,4)$ & 6,1 & & 28,6 & & 57,1 & & 28,6 & \\
\hline \multicolumn{10}{|l|}{ Circunferência da cintura } \\
\hline Adequada & $520(67,9)$ & 15,8 & 0,006 & 50,6 & $<0,001$ & 35,9 & 0,003 & 40,1 & 0,003 \\
\hline Aumentada/Muito aumentada & $246(32,1)$ & 8,6 & & 27,6 & & 47,1 & & 28,9 & \\
\hline \multicolumn{10}{|l|}{ Diabetes } \\
\hline Sim & $43(5,4)$ & 9,3 & 0,446 & 21,4 & 0,005 & 27,9 & 0,115 & 23,3 & 0,085 \\
\hline Não & $754(94,6)$ & 13,3 & & 43,2 & & 40,0 & & 36,2 & \\
\hline \multicolumn{10}{|l|}{ Hipertensão } \\
\hline Sim & $358(45,4)$ & 6,7 & $<0,001$ & 34,9 & $<0,001$ & 36,9 & 0,228 & 31,7 & 0,033 \\
\hline Não & $431(54,6)$ & 18,4 & & 48,1 & & 41,2 & & 39,0 & \\
\hline \multicolumn{10}{|l|}{ Artrite } \\
\hline Sim & $13(1,6)$ & 0,0 & 0,383 & 30,8 & 0,573 & 46,1 & 0,612 & 38,5 & 0,779 \\
\hline Não & $784(98,4)$ & 13,3 & & 42,2 & & 39,2 & & 35,4 & \\
\hline \multicolumn{10}{|l|}{ Autopercepção de saúde } \\
\hline Positiva & $356(44,8)$ & 15,5 & 0,080 & 41,6 & 0,750 & 35,3 & 0,037 & 35,5 & 0,952 \\
\hline Negativa & $438(55,2)$ & 11,3 & & 42,7 & & 42,7 & & 35,7 & \\
\hline
\end{tabular}

IMC: índice de massa corporal.

Valor de p: referente ao teste qui-quadrado de Pearson ou Exato de Fisher.

doméstico do que a encontrada para a população da zona rural de Minas Gerais, uma vez que os quilombolas passam maiores períodos fora de casa. Já o percentual de quilombolas ativos no deslocamento foi similar. As comunidades rurais do Brasil apresentam características que se diferem dependendo da região em que estão inseridas, das condições climáticas, do tipo de vegetação e de uma série de outros fatores que determinam as atividades rurais predominantes, situação que pode explicar as diferenças observadas nas prevalências de AF entre essas populações.

Quanto à percepção do ambiente, os quilombolas que relataram sentir-se seguros ao caminhar durante dia e noite na vizinhança foram mais ativos no lazer. Em uma população de nível socioeconômico baixo da região de São Paulo, observou-se que indivíduos com percepção positiva de segurança foram mais ativos no deslocamento 28 , e os ativos no lazer apresentaram maior média no escore de segurança ${ }^{3}$. Amorim et al. 29 , ao estudarem uma população do Sul do Brasil, verificaram associação positiva entre a percepção de crimes na vizinhança e AF de lazer, mas não observaram associação com a percepção de segurança ao caminhar na vizinhança para AF nos domínios de lazer e deslocamento.

Os resultados do presente estudo são semelhantes às conhecidas associações entre fatores sociodemográficos e AF observadas em populações urbanas. Indivíduos do sexo masculino são mais ativos nos domínios do lazer, trabalho e deslocamento $8,9,26,30$, enquanto as mulheres são mais ativas no domínio doméstico ${ }^{8,9,30}$. Observou-se declínio dos níveis de AF de lazer com o aumento da idade 4,8,26. O mesmo padrão foi encontrado para os domínios trabalho, deslocamento e doméstico 8 .

Para estado conjugal, independentemente de sexo conforme análises estratificadas, indivíduos casados foram mais ativos no domínio doméstico, resultado semelhante ao da população rural de Minas Gerais 9. Provavelmente indivíduos casados permaneçam maior período em casa, principalmente aqueles que vivem em ambientes rurais e trabalham com agricultura de subsistência, o que favorece a realização de AF no contexto do domicílio. Por sua vez, já foi constatado que os solteiros são mais ativos no lazer ${ }^{31}$.

Em concordância com estudos anteriores 8,26, a escolaridade apresentou associação positiva 
Tabela 2

Razões de prevalência ajustadas da associação entre atividade física nos domínios lazer, trabalho, doméstico e deslocamento e variáveis do entorno físico e social, demográficas e socioeconômicas, comportamentais e de saúde/doença.

\begin{tabular}{|c|c|c|c|c|c|c|c|c|}
\hline \multirow[t]{2}{*}{ Variável } & \multicolumn{2}{|c|}{ Lazer } & \multicolumn{2}{|c|}{ Trabalho } & \multicolumn{2}{|c|}{ Doméstico } & \multicolumn{2}{|c|}{ Deslocamento } \\
\hline & RP & IC95\% & RP & IC95\% & RP & IC95\% & RP & IC95\% \\
\hline \multicolumn{9}{|c|}{ Bloco 1: entorno físico e social * } \\
\hline \multicolumn{9}{|l|}{ Segurança na vizinhança } \\
\hline Não & 1,00 & & Excluído & & 1,00 & & 1,00 & \\
\hline Sim & 1,54 & 1,$1 ; 2,35$ & & & 0,84 & 0,$70 ; 1,00$ & 1,14 & 0,$94 ; 1,38$ \\
\hline \multicolumn{9}{|c|}{ Bloco 2: demográficas e socioeconômicas ** } \\
\hline \multicolumn{9}{|l|}{ Sexo } \\
\hline Masculino & 3,46 & 2,$30 ; 5,22$ & 2,99 & 2,$47 ; 3,62$ & 0,46 & 0,$38 ; 0,57$ & 2,03 & 1,$67 ; 2,47$ \\
\hline Feminino & 1,00 & & 1,00 & & 1,00 & & 1,00 & \\
\hline \multicolumn{9}{|l|}{ Idade (anos) } \\
\hline $18-29$ & 4,11 & 1,$82 ; 9,28$ & 1,73 & 1,$26 ; 2,38$ & 1,79 & 1,$29 ; 2,50$ & 1,38 & 1,$00 ; 1,91$ \\
\hline $30-39$ & 2,49 & 1,$07 ; 5,80$ & 1,94 & 1,$43 ; 2,38$ & 1,73 & 1,$25 ; 2,40$ & 1,89 & 1,$38 ; 2,57$ \\
\hline $40-49$ & 1,13 & 0,$43 ; 2,96$ & 2,19 & 1,$63 ; 2,95$ & 1,72 & 1,$24 ; 2,39$ & 1,92 & 1,$41 ; 2,62$ \\
\hline $50-59$ & 1,95 & 0,$80 ; 4,73$ & 2,29 & 1,$70 ; 3,10$ & 1,96 & 1,$39 ; 2,74$ & 1,78 & 1,$28 ; 2,48$ \\
\hline 60 ou mais & 1,00 & & 1,00 & & 1,00 & & 1,00 & \\
\hline \multicolumn{9}{|l|}{ Estado conjugal } \\
\hline Nunca foi casado & 1,00 & & 1,00 & & 1,00 & & Excluído & \\
\hline Casado/Em união & 1,22 & 0,$87 ; 1,72$ & 1,11 & 0,$93 ; 1,33$ & 1,29 & 1,$02 ; 1,64$ & & \\
\hline Separado/Divorciado/Viúvo & 1,50 & 0,$83 ; 2,70$ & 0,90 & 0,$64 ; 1,25$ & 0,89 & 0,$62 ; 1,27$ & & \\
\hline \multicolumn{9}{|l|}{ Escolaridade (anos) } \\
\hline Nunca estudou & 1,00 & & 1,00 & & 1,00 & & 1,00 & \\
\hline $1-4$ & 1,33 & 0,$73 ; 2,42$ & 1,36 & 1,$13 ; 1,65$ & 0,99 & 0,$80 ; 1,23$ & 1,03 & 0,$81 ; 1,31$ \\
\hline 5 ou mais & 2,02 & 1,$12 ; 3,66$ & 1,39 & 1,$11 ; 1,73$ & 0,77 & 0,$58 ; 1,02$ & 0,77 & 0,$58 ; 1,03$ \\
\hline \multicolumn{9}{|l|}{ 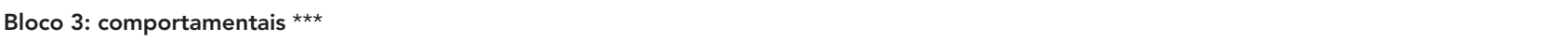 } \\
\hline \multicolumn{9}{|l|}{ Álcool (consumo de risco) } \\
\hline Não & 1,00 & & 1,00 & & 1,00 & & 1,00 & \\
\hline $\operatorname{Sim}$ & 0,94 & 0,$62 ; 1,42$ & 1,17 & 1,$01 ; 1,35$ & 0,78 & 0,$56 ; 1,09$ & 1,15 & 0,$93 ; 1,42$ \\
\hline \multicolumn{9}{|l|}{ Consumo de frutas } \\
\hline Não consome & Excluído & & 1,00 & & 1,00 & & 1,00 & \\
\hline Consome & & & 1,31 & 1,$06 ; 1,62$ & 1,24 & 0,$99 ; 1,54$ & 1,18 & 0,$92 ; 1,51$ \\
\hline \multicolumn{9}{|l|}{ Bloco 4: saúde/doença \# } \\
\hline \multicolumn{9}{|l|}{ Autopercepção de saúde } \\
\hline Positiva & 1,00 & & Excluído & & 1,00 & & Excluído & \\
\hline Negativa & 0,94 & 0,$67 ; 1,31$ & & & 1,22 & 1,$03 ; 1,45$ & & \\
\hline
\end{tabular}

Excluído: variável que apresentou valor de p > 0,20 em análise univariada; IC95\%: intervalo de 95\% de confiança; RP: razão de prevalência.

* Ajustadas entre variáveis do entorno físico e social;

** Ajustadas entre as variáveis do entorno físico e social e demográficas/socioeconômicas;

*** Ajustado entre as variáveis do entorno físico e social, demográficas/socioeconômicas e comportamentais;

\# Ajustado entre as variáveis do entorno físico e social, demográficas/socioeconômicas, comportamentais e saúde/doença.

com AF no lazer, o que pode remeter a um aspecto social, uma vez que a escolaridade pode exercer efeito diferencial sobre o acesso aos recursos de saúde e oportunidades que facilitem a adoção de hábitos saudáveis ${ }^{4}$. Além disso, indivíduos que vivem em vizinhanças com maiores privações socioeconômicas podem estar sujeitos ao menor acesso a espaços de lazer ${ }^{32}$, situação que pode explicar a baixa prevalência de AF no lazer observada na população quilombola.

Também foi observada associação entre escolaridade e AF no trabalho, corroborando o achado do estudo realizado por Pitanga et al. 30, realizado com adultos de etnia negra de Salvador, 
Bahia. Entretanto, nossos resultados são contrários aos observados em trabalho realizado por Florindo et al. 8, no qual indivíduos com maior escolaridade foram menos ativos no ambiente de trabalho. Diferenças no perfil ocupacional podem concorrer para este achado, visto que, em áreas urbanas, grupos mais desfavorecidos economicamente tendem a realizar tarefas com esforço físico laboral maior. Ademais, os quilombolas que nunca estudaram estão sujeitos a realizar menos atividades laborais, pois $48,9 \%$ representam a população mais velha (maiores de 60 anos) e $62 \%$ não trabalham.

Em relação a hábitos e comportamentos, observou-se associação positiva entre consumo de risco para bebidas alcoólicas e AF no trabalho no que se refere aos quilombolas estudados. Martins et al. 33 identificaram essa mesma associação com domínio do lazer na população urbana de Florianópolis, Santa Catarina. Possíveis justificativas para esse quadro é a maior participação em atividades sociais entre os ativos ${ }^{34}$, podendo, ainda, ser resultado do convívio social deste grupo 35 . No presente estudo, foi observado que $41,4 \%$ dos indivíduos ativos no trabalho relataram participar de atividades esportivas ou artísticas em grupo, enquanto, entre os inativos, o percentual foi de apenas $17,7 \%$. Os indivíduos que participavam dessas atividades relataram maior consumo de risco para bebidas alcoólicas (19,9\%) quando comparados aos que não participavam (10,6\%). Portanto, neste estudo, observamos que a população quilombola se assemelha a populações urbanas, possivelmente representando os reflexos de uma globalização dos hábitos e costumes, o que configura um estilo de vida em que fatores de risco coexistem com a prática de AF.

Alguns estudos têm demonstrado associação entre a prática regular de AF e adoção de dietas saudáveis 33,36 , sendo essa associação mais observada no domínio do lazer, o mais explorado 33. Todavia, na presente pesquisa, verificou-se que os quilombolas que relataram consumir frutas pelo menos uma vez ao dia foram mais ativos no domínio do trabalho. Este achado pode, em parte, ser explicado pela maior proporção de lavradores na amostra estudada 12 , levando a um possível acesso diferenciado a esses alimentos.

Associações entre autopercepção de saúde e AF têm sido observadas; pessoas insuficientemente ativas, principalmente no domínio do lazer, tendem a ter pior avaliação da sua saúde 8,37. No entanto, no presente estudo, indivíduos que avaliaram negativamente a saúde foram mais ativos no domínio doméstico, achado que pode, dentre outras possibilidades, refletir as condições de vida precárias em que essas populações estão inseridas. Para justificar essa hipótese, apresen- tamos o argumento de que, em local onde mais de $90 \%$ da população quilombola utilizava poços, cisternas e açudes como forma de obtenção de água para o domicílio 11, o esforço despendido para a realização dessa atividade poderia contribuir para as dores no corpo e, consequentemente, a percepção negativa da saúde relatada por essa população. De fato, foi observada associação positiva entre dores no corpo e percepção negativa da saúde, assim como dores no corpo e AF doméstica. Dos indivíduos ativos no domínio doméstico, 75,3\% relataram dores no corpo de intensidade leve a muito intensa.

Algumas questões metodológicas devem ser consideradas para a interpretação dos resultados obtidos nesta pesquisa. O delineamento transversal impede que se estabeleça a temporalidade entre exposição e desfecho. A utilização de informações autorreferidas para avaliar a prática de AF pode sub ou superestimar a duração e intensidade das atividades. Em adição, ainda que se tenha procurado utilizar o mesmo questionário da PNS, sabe-se que a prevalência de ativos pode variar conforme o instrumento utilizado, dimensões avaliadas e populações entrevistadas. O uso das variáveis de AF na forma contínua é dificultado pela distribuição assimétrica à esquerda dos dados e o excesso de valores zero, em face da baixa prevalência do evento estudado.

A literatura aponta dificuldade de se medir, com a mesma precisão, as atividades realizadas nos diferentes domínios de AF. Em populações urbanas, as atividades realizadas no trabalho e no ambiente doméstico são apontadas como as mais sujeitas a apresentar variação de dia para dia, ou mesmo ao longo do dia, em termos de tipo, intensidade, duração e períodos de pausas 38 . No presente estudo, foram verificados menores valores de CCI e estatística kappa para AF nos domínios doméstico e deslocamento. Uma justificativa talvez seja o fato de que as atividades domésticas e de deslocamento podem se sobrepor ao domínio do trabalho, já que a principal ocupação relatada na população de estudo foi a de lavrador, seguida de empregado doméstico, que exige o uso da força física e está relacionada às tarefas cotidianas do domicílio 9 .

Por outro lado, no presente trabalho, foi utilizada a entrada hierárquica 25,39 , uma estratégia para lidar com um grande número de variáveis explicativas, permitindo que estas sejam posicionadas hierarquicamente por meio do modelo conceitual. A escolha de critérios para a seleção de variáveis não se baseia apenas em critérios estatísticos. A análise dos dados é feita com base em conhecimentos subjacentes que permitem a seleção das variáveis mais fortemente associadas com o desfecho de interesse. 
Os resultados do presente estudo são importantes para o entendimento do comportamento dos diferentes domínios da atividade física no grupo populacional em questão. Os quilombolas apresentaram perfil de AF característico de populações rurais, sendo mais ativos no domínio do trabalho e pouco ativos no lazer. Os determinantes da prática de AF foram semelhantes aos das populações urbanas, e as condições de vulnerabilidade dessa população são evidenciadas ao se identificarem tais fatores.

Estudos que permitam conhecer a situação de vida, prevalências de doenças, hábitos e comportamentos dessas populações são importantes para gerar, de um lado, informações que possam fomentar discussões para políticas de promoção de saúde e, de outro, intervenções de base comunitária, a fim de promover a prática de atividade física, como o Programa Academias da Saúde 40. Sugere-se que elementos culturais, tais como danças e capoeira, sejam estimulados, potencializados pela criação de locais como facilitadores desta ação. Por fim, torna-se imprescindível o investimento na capacitação de profissionais de saúde, para a atenção às principais doenças vivenciadas por esta população relacionadas às práticas culturais, hábitos e comportamentos.

\section{Resumen:}

Este estudio tuvo como objetivo describir la prevalencia y los factores asociados con la actividad física (AF) en los dominios: tiempo libre, trabajo, hogar y desplazamiento entre los quilombolas, tras una encuesta de 797 individuos de edad 18 a 100 años. Con el punto de corte de AF de 150 minutos/semana, se utilizó un modelo de Poisson con entrada jerárquica de las variables. La prevalencia de AF fue: en el trabajo (42,1\%), en el área doméstica (39,3\%), el desplazamiento (35,5\%) y el tiempo libre $(13,1 \%)$. Se asociaron con el AF en el trabajo, el sexo masculino, ser joven, la educación superior, el alcohol y el consumo de frutas; para el dominio hogar: sexo femenino, ser joven, casado y tener una percepción negativa de la salud; para el desplazamiento: sexo masculino y ser joven; y en el tiempo libre: seguridad, sexo masculino, ser joven y educación superior. Concluimos que el AF en quilombolas tenía un perfil característico de las poblaciones rurales, siendo más activo en el trabajo y menos durante el tiempo libre. Los determinantes de la AF fueron similares a los de las poblaciones urbanas.

Actividad Motora; Comunidades Vulnerables; Grupo de Ascendencia Continental Africana;

Factores de Risco

\section{Colaboradores}

V. M. Bezerra e W. T. Caiaffa contribuiram no desenvolvimento do projeto, revisão da literatura, análise dos dados e redação do artigo. A. C. S. Andrade colaborou na revisão da literatura, análise dos dados e revisão da versão final do artigo. C. C. Cesár colaborou na análise dos dados e revisão da versão final do artigo.

\section{Agradecimentos}

A todos os pesquisadores do Projeto COMQUISTA, pela participação na elaboração do projeto, planejamento e supervisão da coleta dos dados. À Capes, pelo financiamento do Doutorado Interinstitucional (DINTER), por meio do Edital 05/2009, Ação Novas Fronteiras. Ao CNPq pela bolsa produtividade em pesquisa da pesquisadora W. T. Caiaffa. 


\section{Referências}

1 World Health Assembly 57.17. Global strategy on diet, physical activity and health. Geneva: World Health Organization; 2004.

2. Hallal PC, Andersen LB, Bull FC, Guthold R, Haskell W, Ekelund U. Global physical activity levels: surveillance progress, pitfalls, and prospects. Lancet 2012; 380:247-57.

3. Florindo AA, Guimarães VV, Farias JCJ, Salvador EP, Sá TH, Reis RS, et al. Validação de uma escala de percepção do ambiente para a prática de atividade física em adultos de uma região de baixo nível socioeconômico. Rev Bras Cineantropom Desempenho Hum 2012; 14:647-59.

4. Bauman AE, Reis RS, Sallis JF, Wells JC, Loos RJ, Martin BW. Correlates of physical activity: why are some people physically active and others not? Lancet 2012; 380:258-71.

5. World Health Organization. Global recommendations on physical activity for health. Geneva: World Health Organization; 2010

6. Hallal PC, Victora CG, Wells JCK, Lima RC. Physical inactivity: prevalence and associated variables in Brazilian adults. Med Sci Sports Exerc 2003; 35:1894-900.

7. Gidlow C, Johnston LH, Crone D, Ellis N, James D. A systematic review of the relationship between socio-economic position and physical activity Health Educ J 2006; 65:338-67.

8. Florindo AA, Guimarães VV, Cesar CL, Barros MB, Alves MC, Goldbaum M. Epidemiology of leisure, transportation, occupational, and household physical activity: prevalence and associated factors. J Phys Act Health 2009; 6:625-32.

9. Bicalho PG, Hallal PC, Gazzinelli A, Knuth AG, Velásquez-Meléndez G. Atividade física e fatores associados em adultos de área rural em Minas Gerais, Brasil. Rev Saúde Pública 2010; 44:884-93.

10. Casa Civil, Presidência da República. Decreto no 4887, de 20 de novembro de 2003. Regulamenta o procedimento para identificação, reconhecimento, delimitação, demarcação e titulação das terras ocupadas por remanescentes das comunidades dos quilombos de que trata o art. 68 do Ato das Disposições Constitucionais Transitórias. http:// www.planalto.gov.br/ccivil_03/decreto/2003/ d4887.htm (acessado em 29/Mai/2012).

11. Bezerra VM, Medeiros DS, Gomes KO, Souzas R, Giatti L, Steffens AP, et al. Inquérito de Saúde em Comunidades Quilombolas de Vitória da Conquista/BA (Projeto COMQUISTA): aspectos metodológicos e análise descritiva. Ciênc Saúde Coletiva 2014; 19:1835-47.

12. Bezerra VM, Andrade ACS, César CC, Caiaffa WT. Comunidades quilombolas de Vitória da Conquis ta, Bahia, Brasil: hipertensão arterial e fatores associados. Cad Saúde Pública 2013; 29:1889-902.

13. Soares DA, Barreto SM. Sobrepeso e obesidade abdominal em adultos quilombolas, Bahia, Brasil. Cad Saúde Pública 2014; 30:341-54.
14. Malta DC, Morais Neto OL, Silva Junior JB. Apre sentação do plano de ações estratégicas para o enfrentamento das doenças crônicas não transmissíveis no Brasil, 2011 a 2022. Epidemiol Serv Saúde $2011 ; 20: 425-38$.

15. Pesquisa Nacional de Saúde. Inquérito Região Integrada do Distrito Federal (RIDE/DF). http://www. pns.icict.fiocruz.br (acessado em 01/Abr/2011).

16. Secretaria de Vigilância em Saúde, Ministério da Saúde. Vigitel Brasil 2011: Vigilância de Fatores de Risco e Proteção para Doenças Crônicas por Inquérito Telefônico. Brasília: Ministério da Saúde 2012. (Série G. Estatística e Informação em Saúde).

17. Landis JR, Koch GG. The measurement of observer agreement for categorical data. Biometrics 1977; 33:159-74.

18. Dumith SC. Proposta de um modelo teórico para a adoção da prática de atividade física. Rev Bras Ativ Fís Saúde 2008; 13:110-20.

19. Saunders JB, Aasland OG, Babor TF, de la Fuente JR, Grant M. Development of the Alcohol Use Disorders Identification Test (AUDIT): WHO Collaborative Project on Early Detection of Persons with Harmful Alcohol Consumption--II. Addiction 1993; 88:791-804

20. WHO Consulation on Obesity. Obesity: preventing and managing the global epidemic. Geneva: World Health Organization; 2000. (WHO Technical Report Series, 894).

21. Lipschitz DA. Screening for nutritional status in the elderly. Primary Care 1994; 21:55-67.

22. Sociedade Brasileira de Cardiologia; Sociedade Brasileira de Hipertensão; Sociedade Brasileira de Nefrologia. VI diretrizes brasileiras de hipertensão. Arq Bras Cardiol 2010; 95(1 Suppl 1):1-51.

23. Chobanian AV, Bakris GL, Black HR, Cushman WC, Green LA, Izzo Jr. JL, et al. Seventh report of the Joint National Committee on Prevention, Detection, Evaluation, and Treatment of High Blood Pressure. Hypertension 2003; 42:1206-52.

24. Bruin A, Picavet HSJ, Nossikov A, editors. Health interview surveys: towards international harmonization of methods and instruments. Copenhagen WHO Regional Office for Europe; 1996. (WHO Regional Publications. European Series, 58).

25. Victora CG, Huttly SR, Fuchs SC, Olinto MTA. The role of conceptual frameworks in epidemiological analysis: a hierarchical approach. Int J Epidemiol $1997 ; 26: 224-7$

26. Knuth AG, Malta DC, Dumith SC, Pereira CA, Morais Neto OL, Temporão JG, et al. Practice of physical activity and sedentarism among Brazilians: results of the National Household Sample Survey-2008. Ciênc Saúde Coletiva 2011; 16:3697-705.

27. Monteiro CA, Conde WL, Matsudo SM, Matsudo VR, Bonsenor IM, Lotufo PA. A descriptive epidemiology of leisure-time physical activity in Brazil, 1996-1997. Rev Panam Salud Pública 2003; $14: 246-54$ 
28. Florindo AA, Salvador EP, Reis RS, Guimarães VV. Percepção do ambiente e prática de atividade física em adultos residentes em região de baixo nível socioeconômico. Rev Saúde Pública 2011; 45:302-10.

29. Amorim TC, Hallal PC, Azevedo MR. Physical activity levels according to physical and social environmental factors in a sample of adults living in south Brazil. J Phys Act Health 2010; 7 Suppl 2:S204-12.

30. Pitanga FJG, Lessa I, Barbosa PSB, Costa MC, Lopes AS. Fatores sociodemográficos associados aos diferentes domínios da atividade física em adultos de etnia negra. Rev Bras Epidemiol 2012; 15:363-75.

31. Dias-da-Costa JS, Hallal PC, Wells JCK, Daltoé T, Fuchs SC, Menezes AMB, et al. Epidemiology of leisure-time physical activity: a population-based study in southern Brazil. Cad Saúde Pública 2005; 21:275-82

32. Hino AA, Reis RS, Sarmiento OL, Parra DC, Brownson RC. The built environment and recreational physical activity among adults in Curitiba, Brazil. Prev Med 2011; 52:419-22.

33. Martins TG, Assis MAA, Nahas MV, Gauche H, Moura EC. Inatividade física no lazer de adultos e fatores associados. Rev Saúde Pública 2009; 43:814-24.

34. Lindström M, Hanson BS, Ostergren PO. Socioeconomic differences in leisure-time physical activity: the role of social participation and social capital in shaping health related behavior. Soc Sci Med 2001; 52:441-51.
35. French MT, Popovici I, Maclean JC. Do alcohol consumers exercise more? Findings from a national survey. Am J Health Promot 2009; 24:2-10.

36. Santos R, Santos MP, Ribeiro JC, Mota J. Physical activity and other lifestyle behaviors in a Portuguese sample of adults: results from the Azorean Physical Activity and Health Study. J Phys Act Health 2009; 6:750-9.

37. Pan SY, Cameron C, Desmeules M, Morrison H, Craig CL, Jiang X. Individual, social, environmental, and physical environmental correlates with physical activity among Canadians: a cross-sectional study. BMC Public Health 2009; 16:9-21.

38. Hallal PC, Reis RS, Parra DC, Hoehner C, Brownson RC, Simoes EJ. Association between perceived environmental attributes and physical activity among adults in Recife, Brazil. J Phys Act Health 2010; 7 Suppl 2:S213-22.

39. Lima S, Carvalho ML, Vasconcelos AG. Proposta de modelo hierarquizado aplicado à investigação de fatores de risco de óbito infantil neonatal. Cad Saúde Pública 2008; 24:1910-6.

40. Ministério da Saúde. Portaria no 719, de 7 de abril de 2011. Institui o Programa Academia da Saúde no âmbito do Sistema Único de Saúde. Diário Oficial da União 2011; 8 abr.

Recebido em 07/Abr/2014

Versão final reapresentada em 05/Dez/2014 Aprovado em 15/Dez/2014 